\title{
Unravelling the atheroprotective mechanisms of LDL immunization
}

A study provides new insights into the mechanisms of immune responses against LDL, showing that anti-LDL immunoreactivity induces antibody-mediated clearance of LDL from blood, increases cholesterol excretion, and reduces vascular inflammation. "We have addressed the paradox of atheroimmunity," remarks lead investigator Göran Hansson. "The local immune response of the atherosclerotic plaque is largely directed against LDL, is pro-inflammatory, and involves mediators known to be pro-atherosclerotic $\left(\mathrm{CD} 4^{+} \mathrm{T}\right.$ helper 1 cells and cytokines such as IFN $\gamma$ ), and yet immunization with LDL elicits a protective immune response that reduces atherosclerosis," he explains.

The research team had previously shown that $\mathrm{CD} 4^{+} \mathrm{T}$ cells recognize oligopeptides of the LDL component apolipoprotein B100 (apoB100).
To assess the immune responses of LDL-reactive $\mathrm{T}$ cells and the effect on atherosclerosis, the investigators used hypercholesterolaemic mice expressing human apoB100, adoptive cell transfer assays, and generated transgenic mice in which the majority of $\mathrm{CD} 4^{+} \mathrm{T}$ cells had T-cell receptors that recognized human apoB100. The experiments showed that a subpopulation of LDL-reactive $T$ cells survived clonal selection of self-reactivity in the thymus and could be activated later in life. After activation by antigen recognition in lymphoid organs, a substantial proportion of these LDL-reactive T cells developed into $\mathrm{T}$ follicular helper cells that evoked a strong B-cell response, with production of antibodies against LDL. These anti-LDL antibodies induced LDL clearance from the circulation through immunecomplex formation, lowering plasma

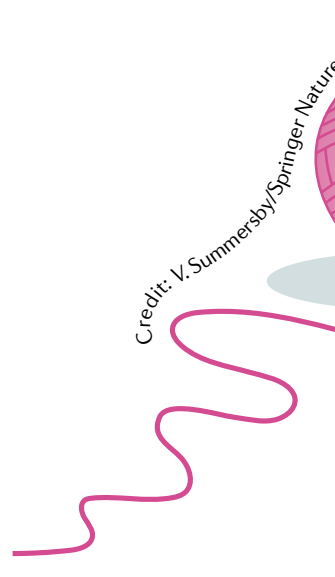

cholesterol levels. LDL autoimmunity was also associated with increased cholesterol excretion in faeces, reduced vascular inflammation, and atheroprotection.

"We show that targeting LDL-reactive T cells can enhance atheroprotective immunity and that vaccination against LDL components may be an attractive way to prevent atherosclerosis," concludes Hansson. The research team is now assessing different apoB100 epitopes and various immunization routes and adjuvants for vaccination to optimize atheroprotective immune responses.

Irene Fernández-Ruiz

against LDL components may be an attractive way to prevent atherosclerosis ?

\section{ACUTE CORONARY SYNDROMES}

\section{Cardiac lymphatics mediate the resolution of inflammation}

Cardiac lymphatics expand in mouse hearts after myocardial infarction (MI); treatment with vascular endothelial growth factor C (VEGF-C) increases this lymphangiogenic response, which improves cardiac function. According to a new study, the expansion of lymphatic vessels after MI promotes heart repair by increasing immune cell clearance.

A previous study suggested that VEGF-C-induced lymphangiogenesis improves cardiac outcome after MI by restoring interstitial fluid equilibrium. Paul Riley and colleagues hypothesized that lymphatic vessels in the heart might also influence the immune response after injury, a phenomenon already described in peripheral tissues during wound healing. "This is an entirely novel finding that was not previously appreciated in the context of heart injury," says Riley. In mice treated with VEGF-C, the number of immune cells in cardiac tissue was reduced 7 days after Ml compared with vehicle-treated mice, but unchanged at earlier time points, when lymphatic sprouting had not yet occurred, suggesting that lymphangiogenesis accelerates the resolution of inflammation after MI.

The constitutive deletion of lymphatic vessel endothelial hyaluronic acid receptor 1 (LYVE1) did not affect cardiac lymphangiogenesis at day 7 after $\mathrm{Ml}$, but Lyve $1^{-1-}$ mice had an increased number of leukocytes in the heart compared with wild-type mice, whereas the number was reduced in mediastinal lymph nodes.

At day 21 after MI, Lyve $1^{--}$mice showed reduced cardiac function and increased scarring compared with wild-type mice. Together, these results indicate that the clearance of immune cells by the lymphatics is dependent on LYVE1 and regulates cardiac outcome after MI.

"This study highlights the cardiac lymphatics as a potential therapeutic target in ischaemic heart disease, but also demonstrates the need to condition the local inflammatory environment in

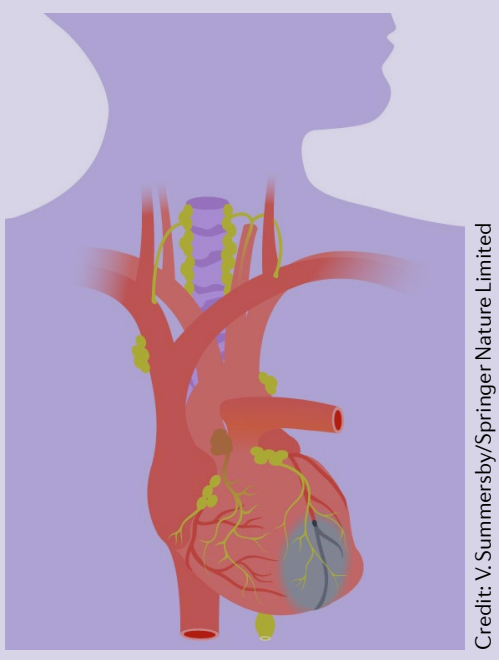

the context of cardiovascular injury as an essential parallel strategy to cell-based repair or regeneration," explains Riley, adding that future studies include the screening of small-molecule inducers of lymphangiogenesis and immune cell clearance as the first stage of a drug-discovery pipeline.

Alexandra Le Bras

ORIGINAL ARTICLE Vieira, J. M. et al. The cardiac lymphatic system stimulates resolution of inflammation following myocardial infarction. J.Clin. Invest. https://doi.org/10.1172/JC197192 (2018) 